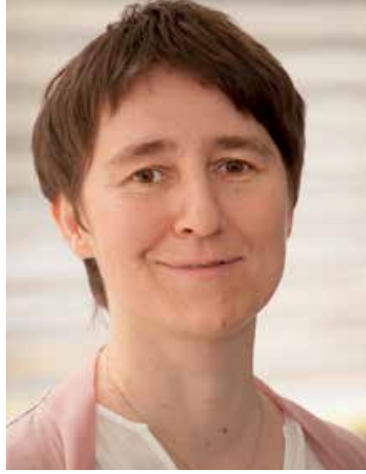

Elke Oldenburg

Redaktionsleitung physiopraxis

elke.oldenburg@thieme.de

\section{Perspektiven für die Zukunft}

„Der Nachwuchs braucht eine Zukunft“, fordert Physiotherapeutin Kristina Jago in ihrem Kommentar auf Seite 9. Sie nahm an einer Podiumsdiskussion zum Thema „Zukunftsperspektiven für Physiotherapeuten“ teil und fragte sich: „Und welches Ziel habe ich?" Gerne würde sie ihre Kompetenzen, die sie während ihres Bachelor- und Masterstudiums erwarb, bei den Patienten anwenden. Doch starre Vorgaben insbesondere durch den Heilmittelkatalog - legen ihr und vielen anderen Therapeuten Fesseln an. Sie will auf Augenhöhe mit den Ärzten aktiv die Versorgungseffizienz erhöhen. Sie ist überzeugt, das spart Kosten. Dafür möchte sie sich engagieren. Dafür braucht es aber auch Freiräume - in Kliniken und von den Krankenkassen.

Rolf Zehnder hat Freiräume für die Physiotherapie geschaffen. Der Schweizer Ökonom ist seit 2008 Direktor des Kantonsspitals Winterthur. Als er 2009 das Spital reorganisierte, hat er im Zuge dessen der Physiotherapie das Vertrauen geschenkt, fachlich und wirtschaftlich eigenständig zu handeln. Die Physiotherapeuten beweisen seither, dass man ihnen vertrauen kann: „Die Physiotherapie ist inzwischen für den Gesamtbehandlungsablauf ein Gewinn und ein ernst genommener Partner“, sagt mir der Klinikdirektor im Interview auf Seite 14. „Dank der Physiotherapie konnten wir die Aufenthaltsdauer reduzieren und haben eine bessere Prä- und Posthospitalisation." Rolf Zehnder würde nicht mehr in das alte System zurückwollen, in dem Physiotherapie Ärzten unterstellt war. Wer am Tropf von anderen hängt, wird nie wirklich eigenständig handeln und beweisen können, was er drauf hat.

Dieses System des Kantonsspitals sollte auch in Deutschland Schule machen! Von mehr Vertrauen in die Physiotherapie profitieren nicht nur Kliniken, sondern auch Krankenkassen finanziell. Zahlreiche Studien belegen: Mit Physiotherapie können Operationen verhindert werden, die teurer wären (○ S. 16). Das spart Kosten in anderen Bereichen. Aber nur, wenn man Physiotherapeuten angemessen vergütet und nicht in Fesseln legt (○ S. 10).
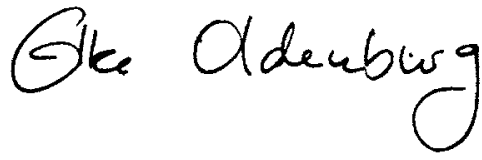

\section{Editorial}

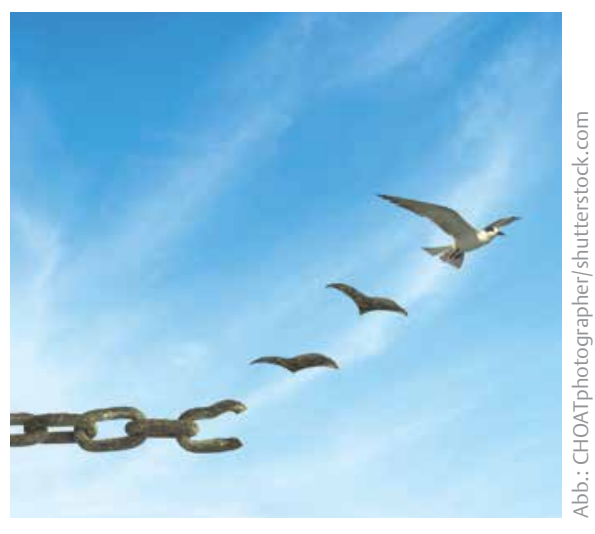

